\title{
Predictors and Clinical Impact of Delayed Stent Thrombosis after Thrombectomy for Acute Stroke with Tandem Lesions
}

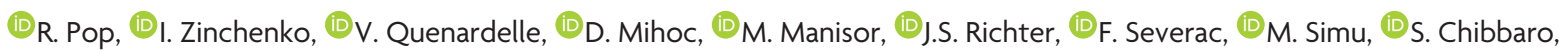 \\ (D) O. Rouyer, 1 V. Wolff, and $\mathbb{D}^{\mathrm{R}}$. Beaujeux
}

\begin{abstract}
BACKGROUND AND PURPOSE: There are very few published data on the patency of carotid stents implanted during thrombectomies for tandem lesions in the anterior circulation. We aimed to communicate our experience of stenting in the acute setting with systematic follow-up of stent patency and discuss predictors and clinical repercussions of delayed stent thrombosis.
\end{abstract}

MATERIALS AND METHODS: We performed a retrospective study of stroke thrombectomies in a single center between January 2009 and April 2018. Patient files were reviewed to extract patient characteristics, procedural details, imaging studies, and clinical information. Predictors of delayed stent thrombosis and clinical outcome at discharge were analyzed using univariate and multivariate analyses.

RESULTS: We identified 81 patients treated for tandem lesions: 63 (77.7\%) atheromas, 17 (20.9\%) dissections, and 1 (1.2\%) carotid web. TICI $2 \mathrm{~b}-3$ recanalization was achieved in $70(86.4 \%)$ cases. Thirty-five patients $(43.2 \%)$ were independent ( $\mathrm{mRS}$ score $\leq 2$ ) at discharge. Among 73 patients with intracranial recanalization and patent stents at the end of the procedure, delayed stent thrombosis was observed in 14 (19.1\%). Among 59 patients with patent stents, 44 had further imaging controls (median, 105 days; range, 2-2407 days) and 1 (1.6\%) had 50\% in-stent stenosis with no retreatment. Stent occlusion rates were $11 / 39$ (28.2\%) for periprocedural aspirin treatment versus $3 / 34$ (8.8\%) for aspirin and clopidogrel $(P=.04)$. Delayed stent thrombosis was independently associated with higher admission NIHSS scores (OR, 1.1; 95\% $\mathrm{Cl}, 1.01-1.28$ ), diabetes (OR, 6.07; 95\% Cl, 1.2-30.6), and the presence of in-stent thrombus on the final angiographic run (OR, 6.2; 95\% Cl, 1.4-27.97). Delayed stent thrombosis (OR, 19.78; 95\% Cl, 2.78-296.83), higher admission NIHSS scores (OR, 1.27, 95\% Cl, 1.12-1.51), and symptomatic hemorrhagic transformation (OR, 23.65; 95\% Cl, 1.85-3478.94) were independent predictors of unfavorable clinical outcome at discharge.

CONCLUSIONS: We observed a non-negligible rate of delayed stent thrombosis with significant negative impact on clinical outcome. Future studies should systematically measure and report stent patency rates.

In: around $15 \%$ of endovascular procedures for anterior circulation stroke, ${ }^{1}$ there is a tight stenosis or occlusion of the cervical carotid artery in addition to the intracranial arterial occlusion. The optimal endovascular management of tandem intra- and extracranial lesions remains subject to debate. The landmark thrombectomy trials either included relatively small numbers of tandem lesions ${ }^{2-4}$ or completely excluded them. ${ }^{5,6}$ Available data

Received October 2, 2018; accepted after revision December 23.

From the Interventional Neuroradiology Department (R.P., D.M., M.M., J.S.R., R.B.), Stroke Unit (I.Z., V.Q., O.R., V.W.), Public Healthcare Department (F.S.), and Neurosurgery Department (S.C.), Strasbourg University Hospitals, Strasbourg, France; Institut Hopitalo-Universitaire Strasbourg (R.P., R.B.), Strasbourg, France; EA3072, FMTS (J.S.R., O.R., V.W.), University of Strasbourg, Strasbourg, France; and Neurology Department (M.S.), Victor Babes University of Medicine, Timisoara, Romania.

Please address correspondence to Raoul Pop, MD, PhD, Service de Neuroradiologie Interventionnelle, UF 6954, Hôpital de Hautepierre, 1 Ave Molière, Strasbourg, France; e-mail: pop.raoul@gmail.com; @RaoulPop25

三 Indicates article with supplemental on-line tables.

http://dx.doi.org/10.3174/ajnr.A5976

mostly consist of retrospective case series published in recent years. $^{7}$

Regardless of technical variations, most groups communicate high recanalization rates with a favorable safety profile for stenting of the extracranial carotid artery. ${ }^{7}$ However, there are very few data available regarding patency rates for the implanted carotid stents and the impact of stent thrombosis on clinical outcome.

Our aim was to communicate our single-center experience in endovascular management of consecutive cases of tandem lesions with systematic follow-up of stent patency and to discuss predictors and clinical repercussions of stent thrombosis.

\section{MATERIALS AND METHODS}

We conducted a retrospective analysis of our prospective data base of acute stroke endovascular procedures between January 1, 2009, and April 1, 2018, using the following inclusion criterion: association of extracranial internal carotid artery occlusion or stenosis of $\geq 70 \%$ using the NASCET criteria and an intracranial 
arterial occlusion in the anterior circulation. Endovascular treatments for complications of surgical carotid endarterectomy were excluded. Images stored on the PACS and radiology reports were reviewed to extract technical details of the endovascular procedure, as well as postprocedural imaging. Patient files were reviewed to extract patient demographics, comorbidities, complications, clinical status at discharge, and clinical follow-up information. The study was approved by the Strasbourg University Hospital's ethics review board. Due to the retrospective nature of the study, the board waived the need for signed informed consent.

\section{Patient Selection and Preprocedural Imaging}

Patients with acute stroke were selected for endovascular procedures using MR imaging, except in case of extreme agitation or absolute contraindications. Patients with favorable profiles for recanalization were selected using clinicoradiologic mismatch (discrepancy between the severity of neurologic deficits and the size of acute ischemic lesion on the diffusion sequence) as well as estimation of leptomeningeal collateral status using FLAIR vascular hyperintensities. ${ }^{8,9}$ We did not use a specific collateral scoring system; vascular hyperintensities were evaluated visually and considered indicative of the presence of ischemic penumbra. Patients with acute infarction in more than two-thirds of the middle cerebral artery territory were generally not considered for treatment. Wake-up strokes and patients with unclear time of onset were considered for treatment if last seen well $<12$ hours before evaluation, using the same imaging-selection criteria.

\section{Endovascular Procedure}

All procedures were performed with the patient under general anesthesia. The strategy did not change during the study period and consisted of an antegrade approach in most cases: stent placement and angioplasty of the proximal occlusion first before addressing the intracranial occlusion. Briefly, a 9F balloon-guide catheter was placed in the distal common carotid artery, and the proximal occlusion was explored with a microcatheter and a 0.014-inch guidewire. If the occlusion could not be crossed using the microcatheter, the system was replaced with a long $4 \mathrm{~F}$ or $5 \mathrm{~F}$ vertebral catheter and a 0.035 -inch guidewire. After crossing the occlusion, we performed a distal angiographic run to assess the distal cervical ICA. Subsequently, a long 0.014 -inch guidewire was advanced into the ICA, and using an exchange maneuver, we placed a carotid stent (usually Wallstent; Boston Scientific, Natick, Massachusetts) covering the lesion and extending to the common carotid artery. The guiding catheter was then advanced inside the stent, and postdilation of the stenosis was performed if needed by means of a $6 \times 20 \mathrm{~mm}$ monorail angioplasty balloon under proximal flow arrest using the balloon of the guiding catheter. The angioplasty balloon was then deflated and removed; the stagnating column of blood was aspirated using a $50-\mathrm{mL}$ syringe before deflation of the balloon-guide catheter. Subsequently, the distal occlusion was treated using a stent retriever, aspiration, or a combination of both methods.

Depending on operator preferences, a minority of cases (mostly carotid dissections) were performed using a retrograde approach. A distal-access catheter or large-bore aspiration catheter was advanced across the proximal lesion, and the distal occlu-

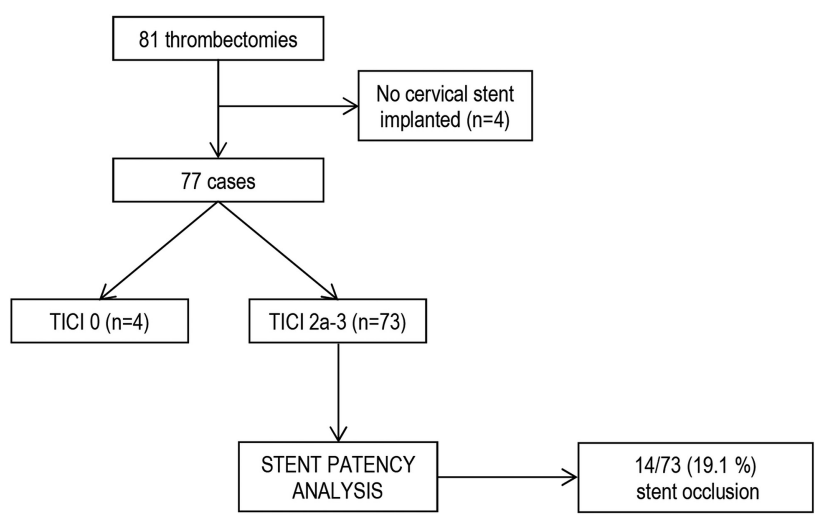

FIG 1. Flow diagram of patient selection for stent-patency analysis.

sion was treated by aspiration or a combination of a stent retriever and distal aspiration. Subsequently, the proximal occlusion was treated with the method previously described, using a long 0.014 inch guidewire advanced through the distal-access catheter.

The antiplatelet and procedural anticoagulation regimen varied across the study period. In the early experience, before carotid stent placement, we administered loading doses of clopidogrel, $300 \mathrm{mg}$ (nasogastric tube), and aspirin, $250 \mathrm{mg}$ (IV); and between 2500 and $4000 \mathrm{U}$ of heparin (IV). Due to an increased rate of hemorrhagic complications, since October 2011, heparin administration was discontinued and the regimen was reduced to IV aspirin $(250 \mathrm{mg})$ with or without a loading dose of clopidogrel (300 mg), depending on operator preferences and case-by-case discussion (estimation of hemorrhagic-transformation risk depending on the size of the acute ischemic lesion and concomitant treatment with IV thrombolysis). If the stent was patent after 24 hours and in the absence of sizeable hemorrhagic transformation, clopidogrel, $75 \mathrm{mg} /$ day, was continued for 3 months in addition to life-long aspirin, $75 \mathrm{mg} /$ day. None of the cases were treated with glycoprotein IIb/IIIa inhibitors.

\section{Postprocedural Imaging and Clinical Follow-Up}

All patients underwent cerebral CT 24 hours postprocedure. Hemorrhagic transformation was evaluated using the European Cooperative Acute Stroke Study criteria. ${ }^{10}$ In addition, for patients with carotid stents, cervical and transcranial Doppler sonography was performed at 24 hours and before discharge to check for stent patency. If a sonographic examination was not feasible at 24 hours, CT angiography of the carotids was performed along with the 24-hour CT examination.

In addition, whenever possible, patients were recalled for additional clinical and carotid sonography examinations between 3 months and 1 year after the initial event.

\section{Evaluation of Delayed Stent Thrombosis}

Delayed stent thrombosis was researched in the subgroup of patients who underwent carotid stent placement, and in whom the procedure resulted in partial or complete recanalization of the cervical and intracranial vasculature. The selection procedure is detailed in Fig 1. Delayed stent thrombosis was defined as carotid stent occlusion diagnosed on follow-up imaging. Predictors of stent thrombosis were researched using univariate and multivariate analyses. 


\section{Clinical Impact of Delayed Stent Thrombosis}

To assess whether delayed stent thrombosis had any repercussions on clinical outcome, we researched predictors of unfavorable clinical outcome at discharge ( $\mathrm{mRS}$ score $>2$ ) within the same subgroup of patients using univariate and multivariate analyses.

\section{Statistical Analysis}

Continuous variables were presented as median and range and compared using the Mann-Whitney $U$ test after assessment of the normality of the distribution. Categoric variables were presented as numbers and percentages and compared using the $\chi^{2}$ test. To assess independent predictors of stent thrombosis and clinical outcome at discharge, we implemented baseline characteristics associated with a $P<.10$ in univariate analyses into backwardstepwise multivariable binary logistic regression models using a removal criterion of $P>.10$. A logistic regression model using the Firth bias reduction method was fitted to handle separation in our data for the clinical outcome at discharge. Results are presented as odds ratios with their 95\% confidence intervals. Statistical data were analyzed using GraphPad Prism, Version 6.0 (GraphPad Software, San Diego, California) and SPSS software, Version 20.0 (IBM, Armonk, New York). The significance level was established at $P<.05$.

\section{RESULTS}

\section{Patient Characteristics}

We identified 81 patients treated for tandem lesions $(77.7 \%$ carotid atheromas, $20.9 \%$ dissections, and 1 case [1.2\%] of a carotid web). Patient demographics and baseline characteristics are detailed in On-line Table 1. The median age was 63 years, and the median admission NIHSS score was 14. Initial imaging consisted of MR imaging in nearly all patients (80/81). Intravenous alteplase was administered in $49.3 \%$ of cases. The median time from symptom onset to femoral puncture was 255 minutes. Of note, $19.7 \%$ of cases were wake-up strokes or with unclear time of onset (in these cases, time when last seen well was used instead of symptom onset).

\section{Thrombectomy Procedure and Outcome}

Technical details of the thrombectomy procedure as well as clinical and imaging outcomes are detailed in On-line Table 2. A carotid stent was implanted in 77 (95\%) patients, of which 42/77 (54.5\%) received periprocedural aspirin (250 mg IV) and 35/77 $(45.4 \%)$ received aspirin and clopidogrel (300 mg via a nasogastric tube). Most patients (83.9\%) were treated using an antegrade approach. The median procedural time was 80 minutes; intracranial circulation TICI 2b-3 recanalization was achieved in $86.4 \%$ of cases. Symptomatic hemorrhagic transformation occurred in $6.1 \%$ of cases. Eight patients $(9.8 \%)$ died during the initial hospitalization. Good clinical outcome ( $\mathrm{mRS} \leq 2$ ) was observed in $43.2 \%$ of patients at discharge. Follow-up was available in $60 / 81$ patients (including deceased patients); after a median interval of 10 months, (range 1-78), $61.6 \%$ of patients had mRS $\leq 2$.

\section{Delayed Stent Thrombosis}

A subgroup of 73 patients had patent carotid stents and partial/ complete intracranial recanalization achieved at the end of the thrombectomy procedure (see Fig 1 for subgroup selection). Cervical imaging at 24 hours consisted of Doppler sonography for $64 / 73$ patients $(87.6 \%)$ and CT angiography for $9 / 73$ patients $(12.3 \%)$.

Delayed stent thrombosis was observed in 14/73 (19.1\%). In most cases (13/14), thrombosis occurred in the first 24 hours; in 1 patient, the stent thrombosed 5 days after the procedure despite double-antiplatelet therapy with aspirin and clopidogrel. Testing of clopidogrel resistance was not performed.

Initially, none of the 14 cases of stent thrombosis were associated with intracranial re-embolization, and imaging demonstrated collateral flow to the MCA via the anterior and/or posterior communicating arteries. However, in 5/14 cases (35.7\%), transcranial Doppler detected lower flow velocities in the MCA compared with the contralateral side, suggestive of insufficient collateralization. Subsequently, in 1 additional patient (1/14, $7.1 \%)$, the MCA reoccluded at 5 days and remained occluded on further follow-up.

On clinical examination, only $3 / 14$ (21.5\%) patients presented with a clear aggravation of neurologic deficits that could be attributed to stent occlusion. They all had reduced MCA flow velocities on transcranial Doppler compared with contralateral side.

Among the 59 patients with patent stents, further imaging follow-up was available for 44 patients (median, 105 days; range, 2-2407 days). One patient (1.6\%) had 50\% in-stent stenosis; there were no retreatments. Among the 14 patients with occluded stents, further stent patency follow-up was available for 11 cases (median, 124 days; range, 5-371 days). The stents remained occluded in all cases.

Stent occlusion rates were significantly higher $(P=.04)$ for patients who received aspirin alone $(11 / 39,28.2 \%)$ compared with aspirin and clopidogrel $(3 / 34,8.8 \%)$.

Univariate analysis of predictors for delayed stent thrombosis is presented in On-line Table 3. In backward stepwise multivariable analysis (Table), delayed stent thrombosis was independently associated with a higher admission NIHSS score (OR per 1-point increase, 1.1; 95\% CI, 1.01-1.28), diabetes (OR, 6.07; 95\% CI, 1.2-30.6), and the presence of in-stent thrombus on the final angiographic run (OR, 6.2; 95\% CI, 1.4-27.97).

Administration of intravenous thrombolysis before thrombectomy was not associated with a significantly reduced rate of stent thrombosis in univariate or multivariate analyses.

\section{Impact of Stent Thrombosis on Clinical Outcome}

Within the same subgroup of 73 patients, 34 (46.5\%) had good clinical outcome at discharge. Among patients with delayed stent occlusion, only $1(7.1 \%)$ was independent at discharge, compared with $33(55.9 \%)$ cases with patent stents $(P=.001)$. The distribution of $\mathrm{mRS}$ scores for both groups is detailed in Fig 2. Univariate analysis of predictors for clinical outcome is presented in On-line Table 4. In backward stepwise multivariable analysis (Table), delayed stent thrombosis (OR, 19.78; 95\% CI, 2.78-296.83), higher admission NIHSS score (OR per 1-point increase, 1.27; 95\% CI, $1.12-1.51$ ), and symptomatic hemorrhagic transformation (OR, 23.65; 95\% CI, 1.85-3478.94) were independently associated with unfavorable clinical outcome at discharge. 
Multivariable regression analysis of predictors for delayed stent thrombosis and clinical outcome at discharge $\mathrm{e}^{\mathrm{a}}$

\begin{tabular}{lcr}
\multicolumn{1}{c}{ Predictors } & OR (95\% Cl) & $\begin{array}{c}P \\
\text { Value }\end{array}$ \\
\hline Delayed stent thrombosis & & \\
Admission NIHSS (per 1-point increase) & $1.1(1.01-1.28)$ & .03 \\
Diabetes & $6.07(1.2-30.6)$ & .02 \\
In-stent thrombus on final angiographic run & $6.2(1.4-27.97)$ & .01 \\
Unfavorable clinical outcome at discharge (mRS $>2)^{\mathrm{b}}$ & & .001 \\
Delayed stent thrombosis & $19.78(2.78-296.83)$ & $<.001$ \\
Admission NIHSS (per 1-point increase) & $1.27(1.12-1.51)$ & .012 \\
Symptomatic hemorrhagic transformation & $23.65(1.85-3478.94)$ & \\
\hline
\end{tabular}

${ }^{a}$ Candidate predictors for delayed stent thrombosis were the following: antiplatelet treatment (aspirin vs aspirin and clopidogrel), admission NIHSS, diabetes, diffusion ASPECTS of $<7$, visualization of in-stent thrombus on final angiographic run, presence of cervical thrombus distal to the proximal lesion, and time from onset to recanalization. Candidate predictors for clinical outcome at discharge were the following: delayed stent thrombosis, admission NIHSS, location of distal occlusion (M2 versus ICA/M1), presence of cervical thrombus distal to the proximal lesion, diffusion ASPECTS of $<7$, symptomatic hemorrhagic transformation, and time from onset to recanalization.

${ }^{b}$ Because none of the patients with good clinical outcome had symptomatic hemorrhagic transformation, a logistic regression model using the Firth bias reduction method was fitted to handle separation in our data for the clinical outcome at discharge.

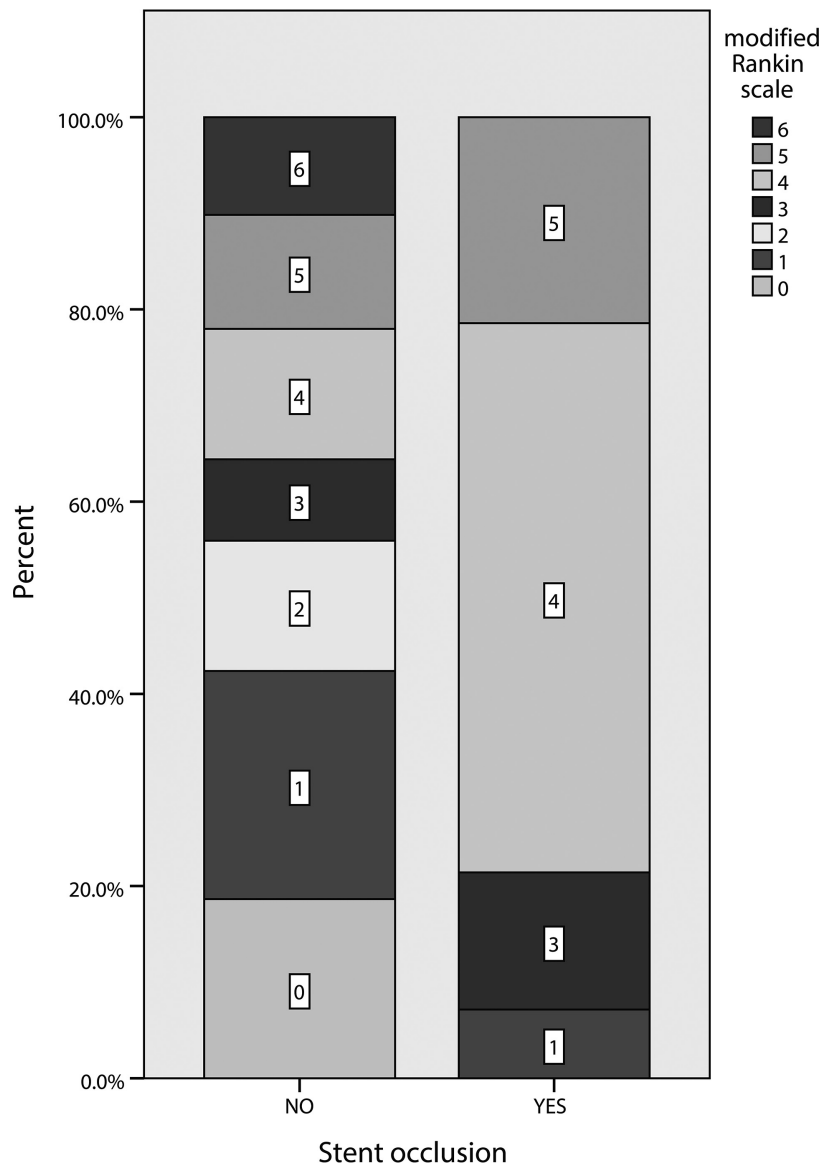

FIG 2. Distribution of $m R S$ scores at discharge in patients with patent-versus-occluded carotid stents. Among patients with delayed stent occlusion, only 1 (7.1\%) was independent (mRS $\leq 2$ ) at discharge, compared with $33(55.9 \%)$ patients with patent stents $(P=.001)$.

An illustrative case of delayed stent thrombosis from this series is presented in Fig 3.

\section{DISCUSSION}

Our study of endovascular treatment for 81 consecutive patients with tandem lesions provides the largest single-center series reported in the literature. By performing systematic imaging fol- low-up of stent patency, we observed a non-negligible rate of delayed stent thrombosis with a significant impact on clinical outcome.

Numerous retrospective case series of endovascular management for tandem lesions have been published in recent years. The data are synthetized in 2 recently published meta-analyses. ${ }^{7,11}$ Most articles reported high recanalization rates with different technical variations of the procedure and identified predictors of successful recanalization and/or good clinical outcome. Surprisingly, there was very little information on the outcome of implanted carotid stents.

In many publications, ${ }^{12-21}$ there is no mention of postprocedural stent patency. Other groups communicate partial data: Sadeh-Gonik et al $^{11}$ studied 43 patients; they reported 1 delayed stent thrombosis of 8 cases with available imaging follow-up (12.5\%). Lockau et $\mathrm{al}^{22}$ performed imaging controls in 28 of 37 patients; there was delayed stent thrombosis in 6 cases $(16.2 \%)$ and significant stenosis in another $2(5.4 \%)$. Steglich-Arnholm et $\mathrm{al}^{23}$ controlled stent patency for 3 months in 43 of a total 47 patients; 4 (9\%) had occluded stents. Heck et $\mathrm{al}^{24}$ controlled stent patency in 18 of 23 cases and found 1 (5.5\%) delayed stent thrombosis; in 13 patients with follow-up sonography ranging from 90 days to 24 months, there were no subsequent events. In a series of 24 cases, Cohen et $\mathrm{al}^{25}$ reported 4 readmissions for new cerebrovascular $(n=2)$ or cardiovascular events $(n=2)$; the stents were patent in all 4 patients. Stent thrombosis rates in these articles are lower than the ones observed in our series, but their data concern only a proportion of the total number of patients. We have shown, in our series, that in most cases $(11 / 14,78.5 \%)$, stent thrombosis was not associated with overt aggravation of neurologic deficits; clinical examination alone therefore seems to be insufficient for detection of stent thrombosis. In the absence of systematic imaging controls of stent patency in the reported series, their real stent thrombosis rates remain unknown.

We identified a single article ${ }^{26}$ reporting 24 -hour imaging follow-up of stent patency for all 77 patients, with only 1 (1.2\%) thrombosed stent. The long-term (30 days or later) in-stent restenosis rate was $2 / 27(7.4 \%)$ in patients with available follow-up imaging. Of note, patients in this series received either epifibatide or double antiplatelet therapy with clopidogrel, $600 \mathrm{mg}$, and aspirin, $325 \mathrm{mg}$, in addition to systemic heparinization. Hemorrhagic transformation occurred in $10.4 \%$ of cases.

Several articles discussed intraprocedural stent thrombosis. Mpotsaris et $\mathrm{al}^{27}$ reported periprocedural in-stent thrombosis in $4 / 63(6 \%)$ procedures. Rangel-Castilla et $\mathrm{al}^{28}$ observed 2/45 $(4.4 \%)$ cases, both resolved with infusion of epifibatide. In a series of 47 patients, Steglich-Arnholm et $\mathrm{al}^{23}$ had 8 (17\%) cases with acute stent thrombosis during thrombectomy, 7 of which resolved with local administration of glycoprotein IIb/IIIa inhibitor; in 1 patient, recanalization was not attempted because of excellent collateral flow and complete intracranial recanalization. 


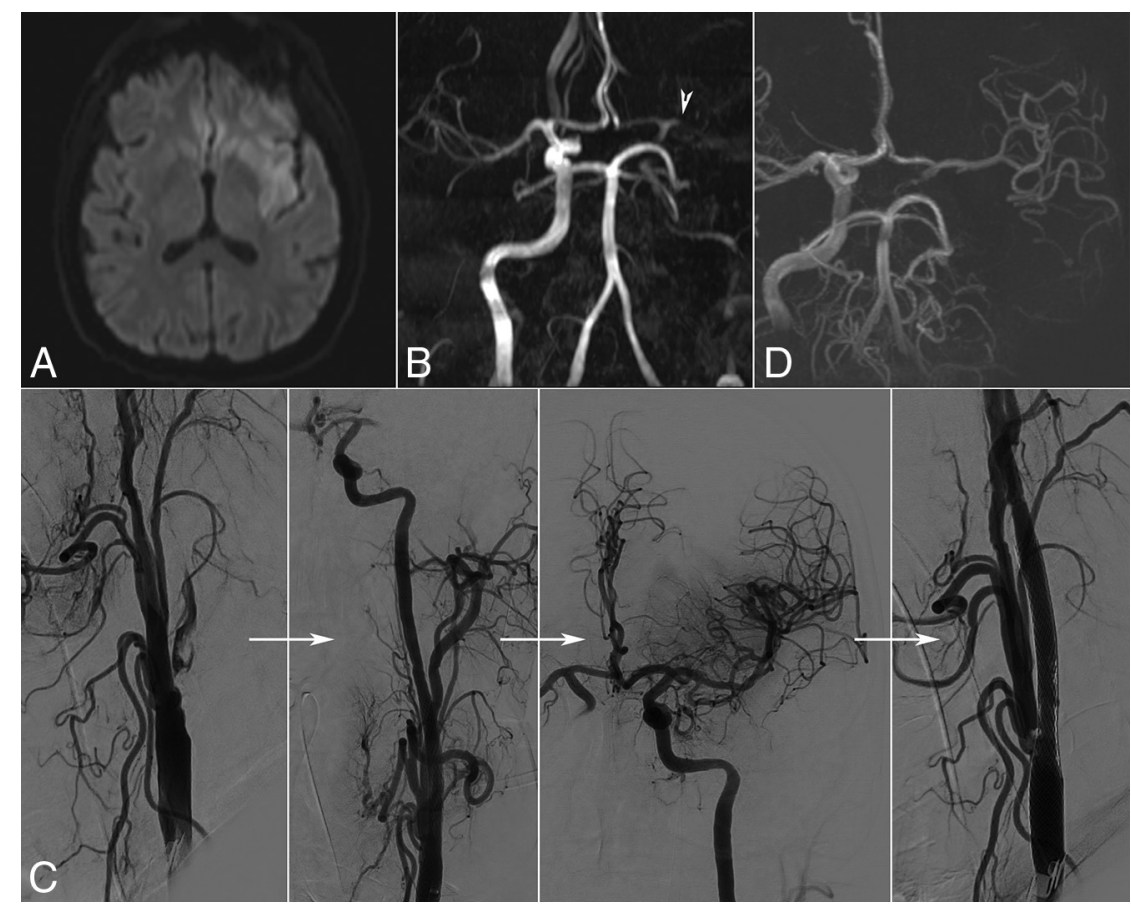

FIG 3. Illustrative case. A patient in his sixties with a history of type 2 diabetes, severe chronic obstructive pulmonary disease, and siderosis was found by his wife hemiplegic and aphasic on wake-up. Initial examination showed depressed consciousness (Glasgow Outcome Score, 7) and signs of respiratory failure for which orotracheal intubation was necessary. Emergency MR imaging showed a relatively small acute ischemic lesion in diffusion imaging $(A)$ not visible in FLAIR imaging, as well as occlusion of the left internal carotid and middle cerebral (arrowhead) arteries $(B)$. Given the important clinicoradiologic mismatch, we proceeded to thrombectomy (C). There was calcified atheroma at the origin of the ICA with floating thrombus. An IV bolus of aspirin, 250 $\mathrm{mg}$, was administered, and a $9 \times 50 \mathrm{~mm}$ Wallstent was deployed and postdilated with a $6 \times 20$ $\mathrm{mm}$ balloon. Then the MCA occlusion was treated by 2 passages of a stent retriever with $\mathrm{TICl} 3$ recanalization. The final cervical angiographic run showed excellent patency of the carotid stent with images suggestive of plaque protrusion but no in-stent thrombus. However, the next day, Doppler sonography demonstrated stent occlusion. The patient remained intubated, with signs of right hemiplegia. Because repeat MR imaging $(D)$ showed flow across the anterior communicating artery and patency of the left MCA, recanalization was not attempted. The patient remains dependent with an NIHSS score of 9 and mRS score of 4 at 3-month follow-up. venous glycoprotein IIb/IIIa inhibitors), differences of implanted stents (varying percentages of metallic surfaces, mesh size, closed- or open-cell design, stent length), subnominal or nominal diameter dilation, and use of overlapping stents.

Third, there seems to be a link between the occurrence of intraprocedural thrombosis and subsequent patency. Intuitively, the underlying pathophysiologic process is the same and is initiated as soon as the stent is implanted. Not surprisingly, we found that visualization of in-stent thrombus on the final angiographic run was an independent predictor of delayed thrombosis. This is concordant with the observation of Steglich-Arnholm et al, ${ }^{23}$ in which all patients with occluded stents at follow-up had also experienced partial or complete stent thrombosis during thrombectomy.

Subsequently, it seems that the risk of thrombosis is highest in the first 24 hours. In our series, almost all (13/14) stent occlusions were diagnosed at 24 hours. Similar results have been reported, ${ }^{24}$ but the number of studied cases is clearly insufficient to draw a conclusion. Given the negative impact of stent thrombosis on clinical outcome, it would seem reasonable to perform more frequent controls of stent patency during the first 24 hours, especially in cases with additional risk factors for stent thrombosis.
Yoon et $\mathrm{al}^{29}$ observed 1 case $(2.2 \%)$ of acute stent thrombosis in a series of 47 patients. Lockau et $\mathrm{al}^{22}$ had $3 / 37(8.1 \%)$ acute stent occlusions during the procedure: One was recanalized by aspiration and balloon angioplasty. In the 2 other cases, recanalization attempts remained unsuccessful, but there was sufficient crossflow from the contralateral site. In our series, 3 cases $(3 / 73,4.1 \%)$ of intraprocedural stent thrombosis were treated successfully with aspiration using a large-bore $6 \mathrm{~F}$ intracranial aspiration catheter or a guiding catheter.

Several conclusions can be drawn from the available literature. First, because the data are clearly insufficient, there is a clear need for systematic follow-up of stent patency in all future case series or prospective studies. This will provide more robust evidence, which can be used to refine the technical details of the endovascular procedure and periprocedural medication, to reduce stent thrombosis rates. In addition, we have shown that delayed stent thrombosis is an independent predictor of unfavorable clinical outcome. Incorporating stent patency data in future studies could improve understanding of clinical outcomes.

Second, the reported stent thrombosis rates were highly variable. There are several causative factors: variability of the procedural antiplatelet protocol (ranging from rectal aspirin to intra-

\section{Intervention for Occluded Carotid Stent}

Once the diagnosis of delayed stent thrombosis has been made, the decision to attempt recanalization can be problematic. In our experience, stent occlusion was not associated with distal re-embolization in the intracranial branches. Initial CT angiography or transcranial sonography demonstrated collateral flow in the MCA through the anterior and/or posterior communicating arteries. In addition, only $3 / 14(21.5 \%)$ patients had clear aggravation of neurologic deficits that could be attributed to stent occlusion. The main procedural risk is distal intracranial embolization during carotid recanalization attempts.

To avoid this clinical dilemma and in light of the clear association between stent thrombosis and unfavorable clinical outcome observed in our series, it seems justified to make every possible effort to prevent delayed stent thrombosis. This involves administering dual-antiplatelet treatment whenever possible, angiographic surveillance of the stent at the end of the thrombectomy for 5-10 minutes, and specific treatment of in-stent thrombus (either by thromboaspiration or administration of glycoprotein IIb/IIIa inhibitors). 


\section{Predictors of Stent Thrombosis}

There was a relatively high rate of delayed stent thrombosis in this study. We believe this is because more than half of the patients with stents $(42 / 77,54.5 \%)$ received a single antiplatelet agent (aspirin) during the first 24 hours.

In addition, most patients in this series were treated using long 50-mm Wallstent stents. In comparison with open-cell designs, the mesh size is smaller and the percentage of metal coverage is higher; these features offer better plaque impaction but are also more thrombogenic in an acute setting.

Delayed stent occlusion was more frequent in patients with diabetes. The association between diabetes and higher rates of stent restenosis and occlusion has been extensively documented in the cardiology literature. ${ }^{30}$ Moreover, diabetes can be associated with an accelerated platelet turnover time, which leads to reduced efficacy of aspirin treatment. ${ }^{31}$ The circulating quantity of new uninhibited platelets rises more rapidly; thus, platelet aggregation returns to normal more rapidly after aspirin administration. To counter this phenomenon, we can speculate that patients with diabetes may need a second dose of aspirin in the first 24 hours, however, further research is needed to balance efficacy versus the added risk of hemorrhagic transformation.

Patients with high NIHSS scores on initial presentation were also more likely to experience delayed stent thrombosis in this series. We can hypothesize that a larger volume of hypoperfused brain leads to decreased carotid outflow and thus promotes stent thrombosis, analogous with peripheral vascular interventions.

\section{Limitations}

This study has several limitations. Patients were identified retrospectively in a single center, and most of the procedures were performed using an antegrade strategy and a single type of stent. Because we included patients during $>9$ years, endovascular approaches and periprocedural anticoagulant/antiplatelet regimens were heterogeneous. In addition, none of the patients in this cohort received glycoprotein IIb/IIIa inhibitors; subsequently, we cannot provide information on stent patency rates for this subgroup.

\section{CONCLUSIONS}

By performing systematic follow-up of stent patency in a consecutive series of thrombectomies for anterior circulation tandem lesions, we observed a non-negligible rate of delayed stent thrombosis in cases with patent stents at the end of the procedure. Stent thrombosis was independently associated with unfavorable clinical outcome at discharge. Stent patency seems to be an important end point that needs to be systematically measured and reported in future studies of tandem lesions.

Disclosures: Johann Sebastian Richter-UNRELATED: Travel/Accommodations/ Meeting Expenses Unrelated to Activities Listed: MicroVention, Balt, Medtronic, Stryker, Penumbra.

\section{REFERENCES}

1. Rubiera M, Ribo M, Delgado-Mederos R, et al. Tandem internal carotid artery/middle cerebral artery occlusion: an independent predictor of poor outcome after systemic thrombolysis. Stroke 2006;37:2301-05 CrossRef Medline
2. Berkhemer OA, Fransen PS, Beumer D, et al. A randomized trial of intraarterial treatment for acute ischemic stroke. N Engl J Med 2015; 372:11-20 CrossRef Medline

3. Goyal M, Demchuk AM, Menon BK, et al; ESCAPE Trial Investigators. Randomized assessment of rapid endovascular treatment of ischemic stroke. N Engl J Med 2015;372:1019-30 CrossRef Medline

4. Jovin TG, Chamorro A, Cobo E, et al; REVASCAT Trial Investigators. Thrombectomy within $\mathbf{8}$ hours after symptom onset in ischemic stroke. N Engl J Med 2015;372:2296-306 CrossRef Medline

5. Saver JL, Goyal M, Bonafe A, et al; SWIFT PRIME Investigators. Stent-retriever thrombectomy after intravenous t-PA vs. t-PA alone in stroke. $N$ Engl J Med 2015;372:2285-95 CrossRef Medline

6. Bracard S, Ducrocq X, Mas JL, et al; THRACE investigators. Mechanical thrombectomy after intravenous alteplase versus alteplase alone after stroke (THRACE): a randomised controlled trial. Lancet Neurol 2016;15:1138-47 CrossRef Medline

7. Wilson MP, Murad MH, Krings T, et al. Management of tandem occlusions in acute ischemic stroke: intracranial versus extracranial first and extracranial stenting versus angioplasty alone: a systematic review and meta-analysis. J Neurointerv Surg 2018;10: 721-78 CrossRef Medline

8. Legrand L, Tisserand M, Turc G, et al. Do FLAIR vascular hyperintensities beyond the DWI lesion represent the ischemic penumbra? AJNR Am J Neuroradiol 2015;36:269-74 CrossRef Medline

9. Legrand L, Tisserand M, Turc G, et al. Fluid-attenuated inversion recovery vascular hyperintensities-diffusion-weighted imaging mismatch identifies acute stroke patients most likely to benefit from recanalization. Stroke 2016;47:424-27 CrossRef Medline

10. Hacke W, Kaste M, Bluhmki E, et al; ECASS Investigators. Thrombolysis with alteplase 3 to 4.5 hours after acute ischemic stroke. N Engl J Med 2008;359:1317-29 CrossRef Medline

11. Sadeh-Gonik U, Tau N, Friehmann T, et al. Thrombectomy outcomes for acute stroke patients with anterior circulation tandem lesions: a clinical registry and an update of a systematic review with meta-analysis. Eur J Neurol 2018;25:693-700 CrossRef Medline

12. Behme D, Mpotsaris A, Zeyen P, et al. Emergency stenting of the extracranial internal carotid artery in combination with anterior circulation thrombectomy in acute ischemic stroke: a retrospective multicenter study. AJNR Am J Neuroradiol 2015;36:2340-45 CrossRef Medline

13. Maurer CJ, Joachimski F, Berlis A. Two in one: endovascular treatment of acute tandem occlusions in the anterior circulation. Clin Neuroradiol 2015;25:397-402 CrossRef Medline

14. Puri AS, Kühn AL, Kwon HJ, et al. Endovascular treatment of tandem vascular occlusions in acute ischemic stroke. J Neurointerv Surg 2015;7:158-63 CrossRef Medline

15. Spiotta AM, Lena J, Vargas J, et al. Proximal to distal approach in the treatment of tandem occlusions causing an acute stroke. J Neurointerv Surg 2015;7:164-69 CrossRef Medline

16. Fahed R, Redjem H, Blanc R, et al. Endovascular management of acute ischemic strokes with tandem occlusions. Cerebrovasc Dis 2016;41:298-305 CrossRef Medline

17. Grigoryan M, Haussen DC, Hassan AE, et al. Endovascular treatment of acute ischemic stroke due to tandem occlusions: large multicenter series and systematic review. Cerebrovasc Dis 2016;41: 306-12 CrossRef Medline

18. Gory B, Haussen DC, Piotin M, et al; Thrombectomy In TANdem lesions (TITAN) investigators. Impact of intravenous thrombolysis and emergent carotid stenting on reperfusion and clinical outcomes in patients with acute stroke with tandem lesion treated with thrombectomy: a collaborative pooled analysis. Eur J Neurol. 2018; 25:1115-20 CrossRef Medline

19. Lescher S, Czeppan K, Porto L, et al. Acute stroke and obstruction of the extracranial carotid artery combined with intracranial tandem occlusion: results of interventional revascularization. Cardiovasc Intervent Radiol 2015;38:304-13 CrossRef Medline

20. Stampfl S, Ringleb PA, Möhlenbruch M, et al. Emergency cervical internal carotid artery stenting in combination with intracranial 
thrombectomy in acute stroke. AJNR Am J Neuroradiol 2014;35: 741-46 CrossRef Medline

21. Kwak HS, Hwang SB, Jin GY, et al. Predictors of functional outcome after emergency carotid artery stenting and intra-arterial thrombolysis for treatment of acute stroke associated with obstruction of the proximal internal carotid artery and tandem downstream occlusion. AJNR Am J Neuroradiol 2013;34:841-46 CrossRef Medline

22. Lockau H, Liebig T, Henning T, et al. Mechanical thrombectomy in tandem occlusion: procedural considerations and clinical results. Neuroradiology 2015;57:589-98 CrossRef Medline

23. Steglich-Arnholm H, Holtmannspötter M, Kondziella D, et al. Thrombectomy assisted by carotid stenting in acute ischemic stroke management: benefits and harms. J Neurol 2015;262:2668-75 CrossRef Medline

24. Heck DV, Brown MD. Carotid stenting and intracranial thrombectomy for treatment of acute stroke due to tandem occlusions with aggressive antiplatelet therapy may be associated with a high incidence of intracranial hemorrhage. J Neurointerv Surg 2015;7:170-75 CrossRef Medline

25. Cohen JE, Gomori JM, Rajz G, et al. Extracranial carotid artery stenting followed by intracranial stent-based thrombectomy for acute tandem occlusive disease. J Neurointerv Surg 2015;7:412-17 CrossRef Medline
26. Malik AM, Vora NA, Lin R, et al. Endovascular treatment of tandem extracranial/intracranial anterior circulation occlusions: preliminary single-center experience. Stroke 2011;42:1653-57 CrossRef Medline

27. Mpotsaris A, Kabbasch C, Borggrefe J, et al. Stenting of the cervical internal carotid artery in acute stroke management: the Karolinska experience. Interv Neuroradiol 2017;23:159-65 CrossRef Medline

28. Rangel-Castilla L, Rajah GB, Shakir HJ, et al. Management of acute ischemic stroke due to tandem occlusion: should endovascular recanalization of the extracranial or intracranial occlusive lesion be done first? Neurosurg Focus 2017;42:E16 CrossRef Medline

29. Yoon W, Kim BM, Kim DJ, et al. Outcomes and prognostic factors after emergent carotid artery stenting for hyperacute stroke within 6 hours of symptom onset. Neurosurgery 2015;76:321-29 CrossRef Medline

30. Wilson SR, Vakili BA, Sherman W, et al. Effect of diabetes on longterm mortality following contemporary percutaneous coronary intervention: analysis of 4,284 cases. Diabetes Care 2004;27:1137-42 CrossRef Medline

31. Di Minno MN, Lupoli R, Palmieri NM, et al. Aspirin resistance, platelet turnover, and diabetic angiopathy: a 2011 update. Thromb Res 2012;129:341-44 CrossRef Medline 